\title{
Performance Analysis of Adaptive Modulation and Coding Schemes on OFDMA Physical Layer
}

\author{
Sayawu Yakubu Diaba ${ }^{1}$, Theophilus Anafo ${ }^{2}$, Zuberu Abdul-Rahman ${ }^{3}$ \\ Suhum Gariba Zongo, Ghana ${ }^{1}$ \\ Tema, Ghana ${ }^{2}$ \\ Rautkalliontie 4E 01360 Vantaa, Finland ${ }^{3}$
}

\begin{abstract}
The recent demand for higher data rate services from wireless network users is overwhelming. Social media influx as well as the proliferation of broadband enabled smart-phones, tablet computers and other newly improved wireless devices has erupted a new trend in wireless network traffic need where average capacity and speed is no longer appreciable. In order to cope with this trend in traffic requirement, wireless network operators are considering a gradual rollover of an existing third generation $(3 \mathrm{G})$ network to a fourth generation $(4 \mathrm{G})$ network with orthogonal frequency division multiple access (OFDMA) based technologies such as Fourth Generation Long Term Evolution (4G LTE) and Worldwide Interoperability for Microwave Access (WiMAX). This paper is devoted to the performance evaluation of Adaptive Modulation and Coding (AMC) in downlink of an orthogonal frequency division multiple access (OFDMA) network, considering Partial Usage of Sub-channels (PUSC). By using MATLAB Simulink and Origin 61, the performance of Bit Error Rate (BER) and Spectral Efficiency in two channel environments, i.e. non-fading and fading channels were examined. Results suggest better performance for AMC over individual MCS in all channel environments. Moreover, non-fading Addictive White Gaussian Noise (AWGN) channels significantly perform better than fading (Rayleigh and Rician) channels. In Rician channel environment, however, flat fading Rician channels perform better than frequency selective Rician channel which interestingly records a degraded performance against Rayleigh channels.
\end{abstract}

Keywords: third generation (3G), fourth generation (4G), Orthogonal Frequency Division Multiple Access (OFDMA), Long Term Evolution (LTE), Worldwide Interoperability for Microwave Access (WiMAX), Bit Error Rate (BER), Adaptive Modulation and Coding (AMC), Partial Usage of Sub-channels (PUSC), Rayleigh channels and Rician channels

\section{INTRODUCTION}

There has been enormous growth in the are currently in early deployment stage in some and yet to Telecommunication and ICT industry over the past few be deployed in some offer speed from 100Mbps to $1 \mathrm{Gbps}$ decades with mobile and wireless communication providing enormous data rate for bandwidth intensive dominating. Presently, there are over six billion eight services and applications.

hundred million mobile cellular subscribers globally with mobile-broadband subscriptions approaching two billion three hundred million. [1,2]. The integration of voice, video and data, which is providing multimedia services has given rise to the demand for the progressive drift from high capacity voice services to high speed and high capacity data services.

The arrival of sophisticated mobile devices and the tremendous bandwidth requirement of certain services and applications such as video calls, video conferencing, online games and the increased utilization of data websites such as Facebook and You tube ignite the need for higher capacity and higher data rate supported technology [3, 17]. Hence the development of the 4G (4th generation) Orthogonal Frequency division multiple access (OFDMA) based wireless technologies such as Mobile WiMAX (Worldwide Interoperability for Microwave Access) and 3GPP (3rd Generation Partnership Project) LTE (Long Term Evolution) which have been identified as promising, permitting higher data rate in wireless broadband access [5]. 4G networks such as WiMAX, LTE and UMB which

The concept of WiMAX is based on the Open Systems Interconnections (OSI) reference model which has the lowest layer as the physical layer which is based on OFDM technology. It is responsible for the specification of the bandwidth, modulation and coding scheme, data rate, multiplexing, error correction, transmitting data in frames, synchronization between transmitter and receiver and controlling access to shared wireless medium classified under media access control (MAC) layer [4].

The IEEE 802.16 (WiMAX) standard is as a result of the increasing higher capacity and higher speed communication required for video, voice and multimedia to meet the growing effect on how people interact or communicate as well as enjoy their entertainment. WiMAX standards have evolved through the years since its introduction in 2001 with its IEEE 802.16 standard which was also known as fixed WiMAX [8].

Interoperability is the key objective of WiMAX which ensures that, equipment from different vendors interoperates in the framework without difficulty. 
International standards do not define boundaries for subscriber module usage. It supports scalable OFDMA, QPSK, 16QAM and 64QAM with channel bandwidth selection within the range of $1.75 \mathrm{MHz}$ to $20 \mathrm{MHz}[5,7]$. However, signal power loss is prevalent along the propagation path for certain frequencies hence leading to limited coverage. Additionally, dead spot and areas of poor reception caused due to fading and blocking within coverage further reduce the efficiency of this standard. Conversely, one of the salient efforts to solve this problem has been to deploy relatively more base stations (BS) within a geographical area [13]. Resource allocation for both uplink and downlink are controlled by a scheduler in the base station.

The authors of $[10,11]$ stated that, the WiMAX physicallayer is capable of supporting MIMO system which allows the WiMAX architecture to adapt the use of multiple antenna techniques for example beam forming, space time coding and spatial multiplexing

According to [8, 9] the MAC layer of WiMAX supports not only fixed bit rates but also variable bit rates, real-time and non-real-time traffic flows and also supports the best effort data traffic.

The most innovative security features that are presently used wireless access schemes are integrated in Mobile WiMAX for voice, data and multimedia services. Such features comprise, Advance Encryption Standard (AES) based authentication and encryption, Cipher-based Message Authentication Code (CMAC) and Hashed Message Authentication Code (HMAC) based control message protection schemes and Extensible Authentication Protocol (EAP) based authentication. Thus supporting varied user credentials such as digital certificates, username and user password schemes, smart cards and SIM/USIM cards [7].

The above features needs further investigation in terms SNR on BER over various channels.

This paper is structured as follows. The introduction is presented in chapters I. In chapter II the system model is presented, while chapter III presents the methodology. Section IV presents the simulation results and conclusion is drawn in chapter $\mathrm{V}$.

\section{WIMAX OVERVIEW}

The WiMAX Forum Network Group (WiMAX NWG) developed the network architecture of WiMAX to guarantee interoperability among diverse vendors and their broadband equipment [8].

WiMAX (Worldwide Interoperability for Microwave Access) is based on the IEEE 802.16 standard, formulated to offer a shared basis for wireless connectivity in fixed, portable, and mobile environment. WiMAX is a scalable digital wireless access $4 \mathrm{G}$ technology intended to deliver high throughput over long distances "wireless metropolitan area networks" WMAN [5]. As a wireless broadband technology, WiMAX perform similar to Wi-Fi (IEEE 802.11) networks providing QoS (Quality of
Service) and coverage for fixed and mobile networks. It is intended to be a complement or a competitor to cellular technologies such as LTE and UMTS.

Interoperability is the key objective of WiMAX which ensures that, equipment from different vendors interoperates in the framework without difficulty. International standards do not define boundaries for subscriber module usage.

The systems is capable of covering large areas due to various modulation schemes adopted such as QPSK, 16 QAM and 64 - QAM.

It is capable of providing enormous capacity for bandwidth intensive applications compared to Universal Mobile Telecommunication System (UMTS) and Global System for Mobile communication (GSM).

User integrity, authentication and confidentiality are assured through encryption and authentication protocols such as AES adopted by the WiMAX standard. Numerous system designs including Point-to-Point, Point-toMultipoint and Permeating coverage is permissible. It provides communication for different traffic including VoIP, multimedia applications and data, whiles ensuring a higher degree of QoS.

Mobility is the key feature of the IEEE 802.16e and IEEE 802.16j standards due to Scalable Orthogonal Frequency Division Multiple Access (SOFDMA) and Multiple Input and Multiple Output (MIMO) techniques adopted at the physical layer $[5,6,8]$.

\section{PAGE STYLE}

The data bit stream of OFDM is divided into $\mathrm{N}$ data streams using a rate of with each being parallel to each other. i.e., the available spectrum must be divided into several narrow sub-channels. Equalization becomes very simple due to flat fading. Cyclic prefix, which is a copy of last part of the OFDM symbol, is used in our model to mitigate inter-symbol and inter-carrier interference while maintaining orthogonality even though time-dispersive channel is used for communication. This causes the transmitted signal to appear periodic.

At a unique frequency, the individual streams are mapped into tones and combined via IFFT (Inverse Fast Fourier Transform) to produce the signal in time domain

\section{A. OFDMA Transmitter}

At the transmission stage, the signal in low pass is given by:

$$
x(t)=\frac{1}{\sqrt{T_{s}}} \sum_{k=0}^{N-1} s_{k}(n) e^{j 2 \pi f_{0} t}
$$

With $t$ defined within the interval; $n T_{s} \leq t<(n+1) T_{s}$, where $S_{k}$ representing the baseband modulated signal.

The orthogonality of each subcarrier in OFDM according to [15] is given by: 


$$
\frac{1}{T_{s}} \int_{0}^{T_{s}} e^{j 2 \pi\left(f_{k}-f_{0}\right) t} d t=\left\{\begin{array}{l}
1, k=l \\
0, k \neq l
\end{array}\right.
$$

With the minimum spacing given by:

$$
\Delta f=f_{k+1}-f_{k}=\frac{1}{T_{s}}
$$

Hence by substitution, equation one becomes:

$$
x(t)=\frac{1}{\sqrt{T_{s}}} \sum_{k=0}^{N-1} s_{k}(n) e^{j 2 \pi k \frac{t}{T_{s}}}
$$

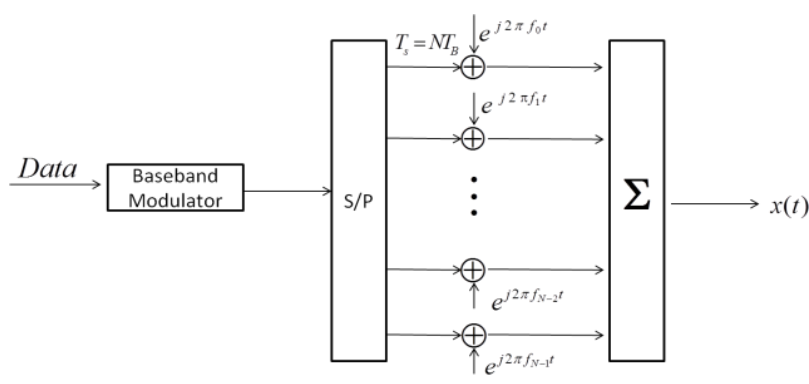

OFDMA Transmitter

Finding the FT (Fourier Transform) of the signal produces the frequency version the baseband signal given by:

$$
\begin{aligned}
& X\left(f_{1}, n T_{s}\right)=\frac{1}{T_{s}} \int_{n T_{s}}^{(n+1) T_{s}} x(t) e^{j 2 \pi f t} d t \\
= & e^{-j \theta_{n}} \sum_{k=0}^{N-1} s_{k}(n) \sin c\left(f T_{s}-k\right)
\end{aligned}
$$

Sampling $X\left(f_{1}, n T_{s}\right)$ at $f=k / T_{\text {produces }} s_{k}(n)$.

Thus:

$$
X\left(\frac{k}{T_{s}}, n T_{s}\right)=s_{k}(n)
$$

Where $k=0,1,2, \ldots \ldots . N-1$

For the channel it is we assumed $h(\tau ; t)$ to be the time invariant response within the interval $0 \leq \tau \leq T_{c p}$ limited to the cyclic prefix length. The received signal $r(t)$ is given by:

$$
r(t)=(h * x)(t)=\int_{0}^{T_{c p}} h(\tau ; t) x(t-\tau) d \tau+z(t)
$$

With $z(t)$ representing additive complex and white Gaussian noise.

\section{B. OFDMA Receiver}

For the receiver model, we assume a filter cache which is matched to the last part of the waveform $\phi_{k}(t)$ given by:

$$
\psi_{k}(t)=\left\{\begin{array}{cc}
\phi_{k}^{*}(T-t), & \left(0 \leq t \leq T-T_{c p}\right) \\
0, & \text { otherwise }
\end{array}\right.
$$

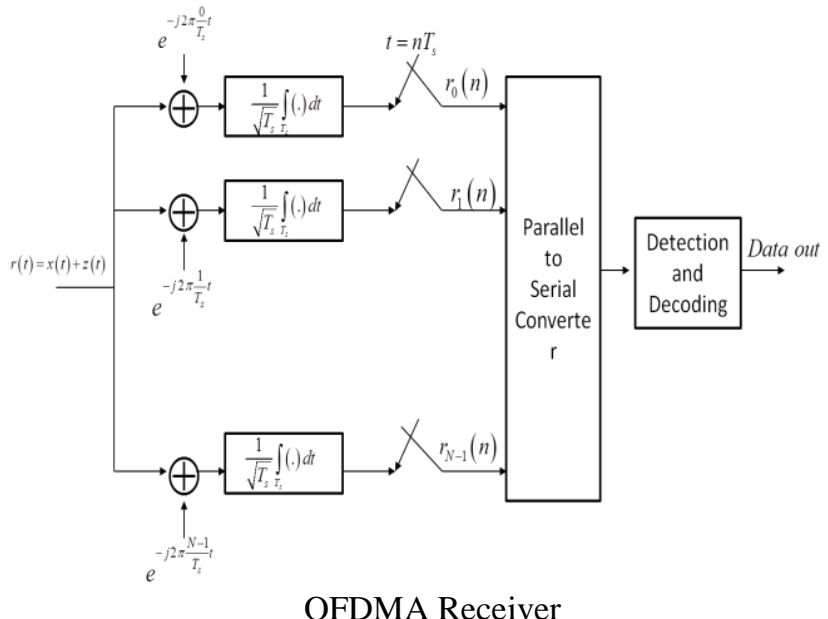

By adding cyclic prefix, the transmitter utilizes rectangular pulse modulated waveforms given by [12]:

$$
\phi_{k}(t)=\left\{\begin{array}{lc}
\frac{1}{\sqrt{T-T_{c p}}} e^{j 2 \pi \frac{W}{N} k\left(t-T_{c p}\right)}, & t \in(0, T) \\
0, & \text { otherwise }
\end{array}\right.
$$

On a carrier frequency $k W / N$, where $N$ represent the number of subcarriers, $W$ represent bandwidth, $t$ represent symbol length and $T_{c p}$ represent the length of cyclic prefix while

$$
T=N / W+T_{c p}
$$

However,

$$
\phi_{k}=\phi_{k}(t-N / W)
$$

When $0<t<T_{c p}$ (i.e. when $t$ is limited to the cyclic prefix)

Therefore the baseband signal for OFDM symbol number $n$ to be transmitted is given by:

$$
x_{n}(t)=\sum_{k=0}^{N-1} i_{k, n} \phi_{k}(t-n T)
$$

With $i_{0, n}, i_{1, n}, i_{2, n}, \ldots \ldots, i_{N-1, n}$ being a set of constellation points complex numbers. For infinite sequence of OFDM symbols $x(t)$ to be transmitted,

$$
x(t)=\sum_{n=-\infty}^{\infty} x_{n}(t)=\sum_{n=-\infty}^{\infty} \sum_{k=0}^{N-1} i_{k, n} \phi_{k}(t-n T)
$$


International Journal of Advanced Research in Computer and Communication Engineering Vol. 4, Issue 12, December 2015

\section{SIMULATION RESULTS}

The simulation model comprises of a downlink PUSC (partial usage of sub-channels) Physical Layer communication between two MS (mobile stations) and a BS (base station) based on the IEEE 802.16j standard. It support all the mandatory modulation and coding schemes offered by the standard, and demonstrate the variable size capability of Embedded MATLAB, Simulink, Communication System Toolbox and DSP System Toolbox [16]

For simplicity, the model is limited to two MS while applying FFT size of 1024. The paper specified 1024 subcarriers out of which 720 are reserved for user data carriage and the remaining $304 \mathrm{kept}$ for pilots and guards. The 720 subcarriers are grouped into 30 sub-channels with each channel holding 24 subcarriers. This allows for efficient allocations of data carriers to different MS with a sub-channel being the smallest unit permissible for allocation.

The model allows for dynamic allocation of the subchannels or frequency resources to MS, thus, the BS is able to dynamically adjust sub-channel allocation to MS1 and MS2 while the system is still running. For instance, sub-channels 10 to 15 allocated to MS1 and sub-channels 16 to 28 allocated to MS2 in one burst can adjust to becoming sub-channels 0 to 10 allocated to MS1 and subchannels 11 to 20 allocated to MS2 in another burst. This initiates variable size signalling since data transmitted is directly proportional to sub-channels allocated.

First, we perform our simulations by transmitting over a non-fading pure AWGN (Additive White Gaussian Noise) channel while adjusting the SNR at various levels. The result of this is demonstrated in figure 4.1 and figure 4.2 for MS1 and MS2 respectively.

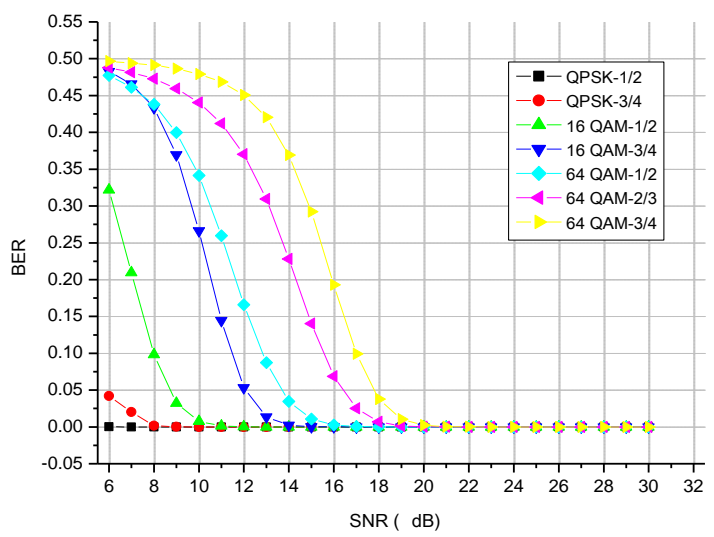

Figure 4.1 Effect of SNR on BER for various MCSs in AWGN channel, (MS1).

The two figures showing the BER performance for MS1 and MS2 state clearly that, the performance improve as SNR values goes high. There is no significant difference between the performance of MS1 and MS2 in AWGN channel without multipath fading. For both MSs, QPSK$1 / 2$ obtain optimum performance for SNR value at $8 \mathrm{~dB}$ and thereafter while 64 QAM-3/4 obtain best performance at $24 \mathrm{~dB}$ SNR value and subsequent values.

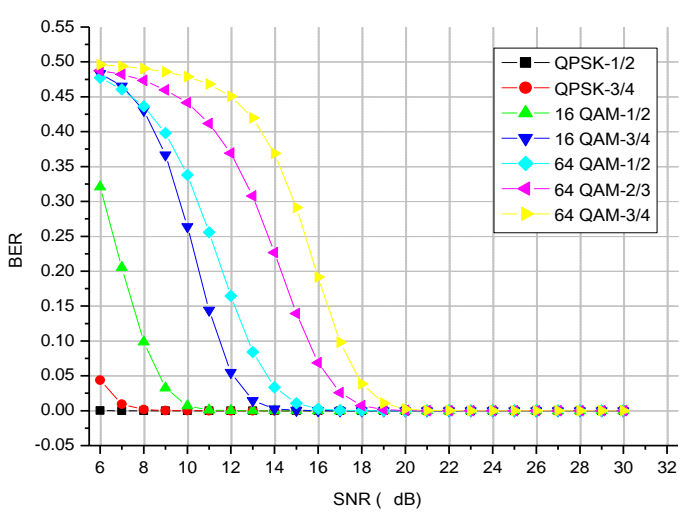

Figure 4.2 Effect of SNR on BER for various MCSs in AWGN channel, (MS2)

Next, a Flat Fading Rician Channel is introduced in AWGN environment while keeping all parameters constant. Figures 4.3 and 4.4 show the BER performance recorded for MS1 and MS2 respectively. It is observed that, Flat Fading Rician channel has a significant effect on the fidelity of the signal the signal received. Higher signal power with respect to the noise factor is required for optimum performance compared to when pure AWGN is considered.

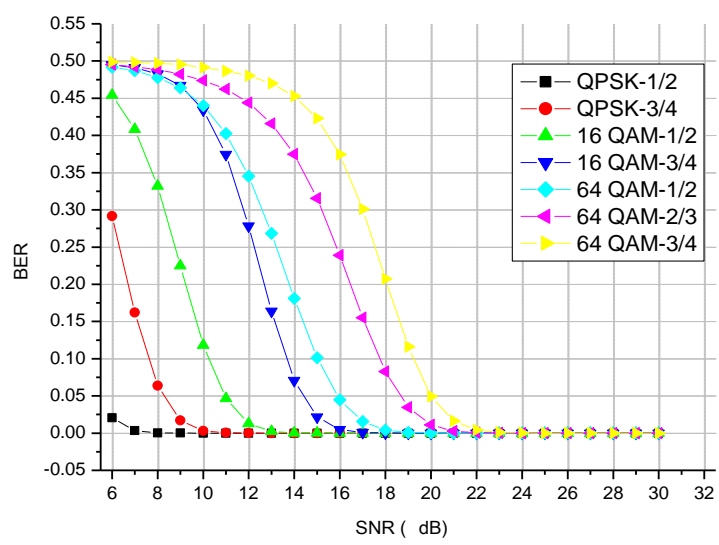

Figure 4.3 Effect of SNR on BER over a Flat Fading Rician + AWGN Channel, (MS1)

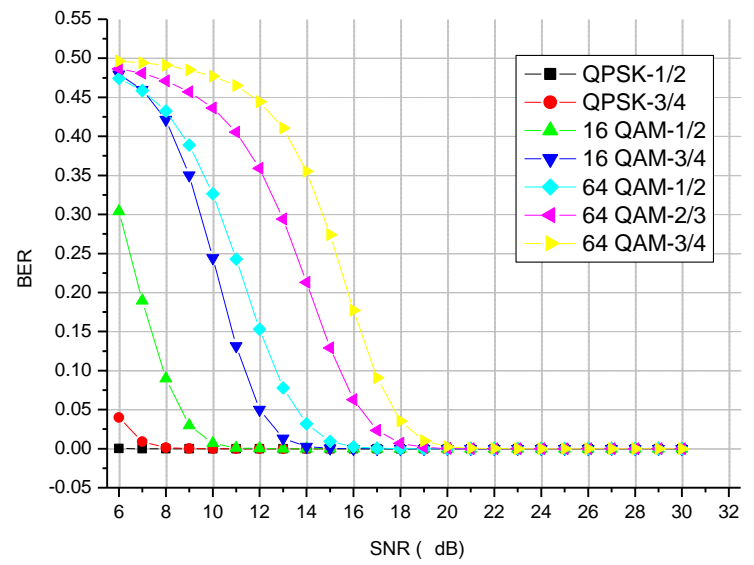

Figure 4.4 Effect of SNR on BER over Flat Fading Rician + AWGN Channel, (MS2)

For MS1, it is realized that, QPSK-1/2 records optimal performance at $10 \mathrm{~dB}$ SNR and beyond while 64 QAM- 
$3 / 4$ records its optimal performance at $27 \mathrm{~dB}$ SNR and beyond. On the hand, for MS2, QPSK recorded maximum performance at $8 \mathrm{~dB}$ SNR while 64 QAM recorded level best performance at $25 \mathrm{~dB}$ SNR which varied marginally with that of AWGN channel.

Figures 4.5 and 4.6 depict the BER performance of Frequency Selective Rician fading channel with AWGN. For MS1, the performance of QPSK-1/2 (least modulation and coding rate) reached level best at $16 \mathrm{~dB}$ SNR value while 64 QAM-3/4 (highest modulation and coding rate) obtained optimal performance at $36 \mathrm{~dB}$ SNR value and thereafter. For MS2, QPSK-1/2 optimal performance at $18 \mathrm{~dB}$ SNR values and 64 QAM-3/4 recorded most favourable performance at as high as $52 \mathrm{~dB}$ values of SNR which does not reach zero BER at this point and beyond. This clearly indicate that, Frequency Selective Rician fading channels offer stiffer opposition to signal transmission compared to Flat fading Rician and AWGN channels.

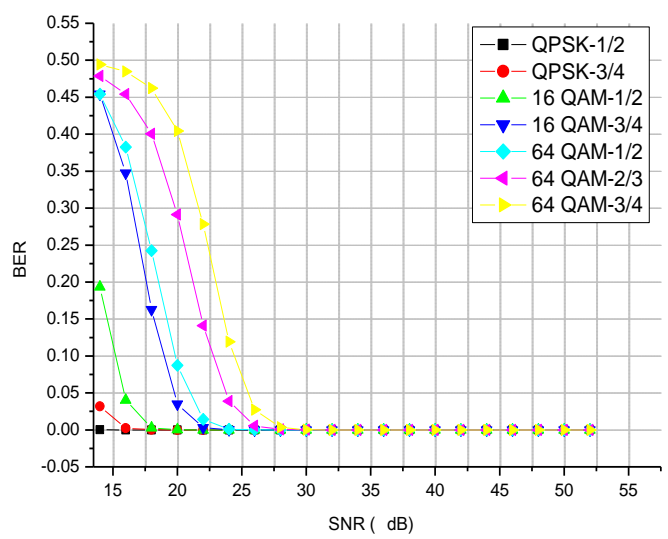

Figure 4.5 Effect of SNR on BER over Frequency Selective Rician + AWGN Channel, (MS1)

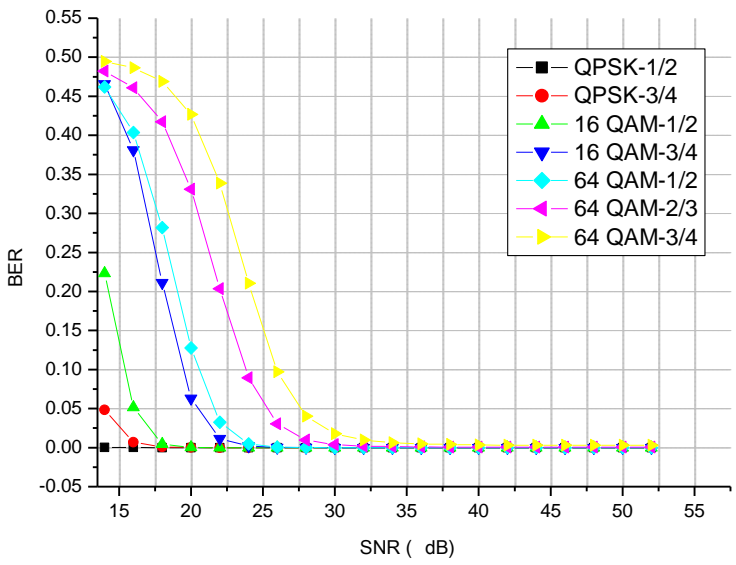

Figure 4.6 Effect of SNR on BER over Frequency Selective Rician + AWGN Channel, (MS2)

BER performance of transmission over Rayleigh fading with AWGN channel is analyzed next. From figure 4.7 which depicts the performance of MS1, QPSK-1/2 (least modulation and coding rate) recorded optimal performance at $8 \mathrm{~dB}$ and higher values of SNR while 64 QAM-3/4 (highest modulation and coding rate) recorded optimal performance with SNR values at $34 \mathrm{~dB}$ and higher.
For MS2 depicted in figure 4.8, as QPSK-1/2 records optimal performance at $8 \mathrm{~dB}$ and higher values of SNR, 64 QAM-3/4 records best level performance which approaches but never reaches zero BER at as high as $40 \mathrm{~dB}$ and higher SNR values. This indicates that, Rayleigh Fading channel requires higher signal power relative to noise for optimum performance compared to Rician and AWGN channels.

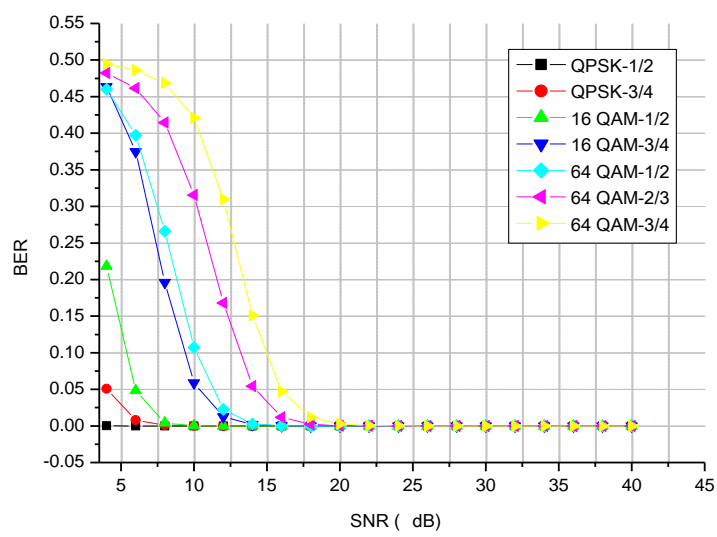

Figure 4.7 Effect of SNR on BER over Rayleigh + AWGN Channel, (MS1)

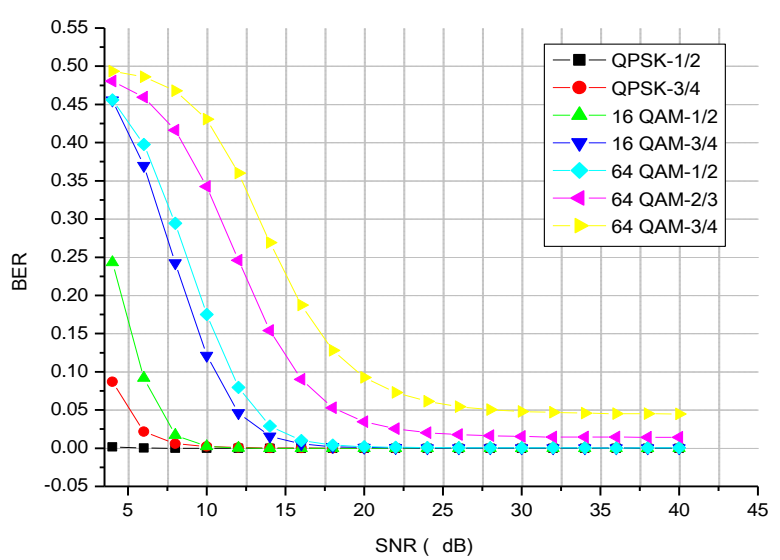

Figure 4.9 Effect of SNR on BER over Rayleigh + AWGN Channel, (MS2)

After examining the BER performance, we investigate the spectral efficiency against increase in SNR for MS1 over the various channels under study. The average spectral efficiency is seen as the number of successful bits transferred per second per hertz of bandwidth [14]. For each MCS, the spectral efficiency is plotted as a function of SNR while determining the values of SNR that record optimal spectral efficiency.

It is evident from figure 4.9 that, the maximum spectral efficiency for AMC over AWGN channel is obtained as $8.789 \mathrm{bps} / \mathrm{Hz}$ at SNR values greater than or equal to $24 \mathrm{~dB}$ However, for individual MCSs depicted in figure 4.10, the maximum spectral efficiency vary increasingly from QPSK-1/2 recording $1.953 \mathrm{bps} / \mathrm{Hz}$ at $8 \mathrm{~dB}$ SNR value to $64 \mathrm{QAM}-3 / 4$ recording $8.789 \mathrm{bps} / \mathrm{Hz}$ at $24 \mathrm{~dB}$ SNR value.

An increase in code rate produced an increase in spectral efficiency as the SNR requirement also goes high. 


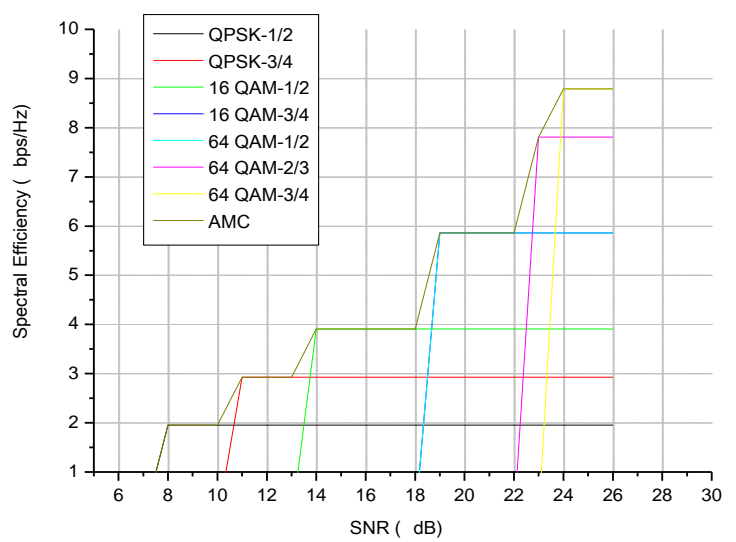

Figure 4.9 Spectral Efficiency versus SNR of AMC over AWGN channel

Figure 4.10 depict the spectral efficiency for various MCS plotted against SNR in AWGN channel. Comparing figures 4.9 and 4.10, it is realized that adopting AMC will produce better spectral efficiency than using individual MCS. Similar investigations and analysis are made for transmission over fading (Rician flat fading, Rician frequency selective and Rayleigh) channels and the results are demonstrated in figures 4.11, 4.12, 4.13, 4.14, 4.15 and 4.16 .

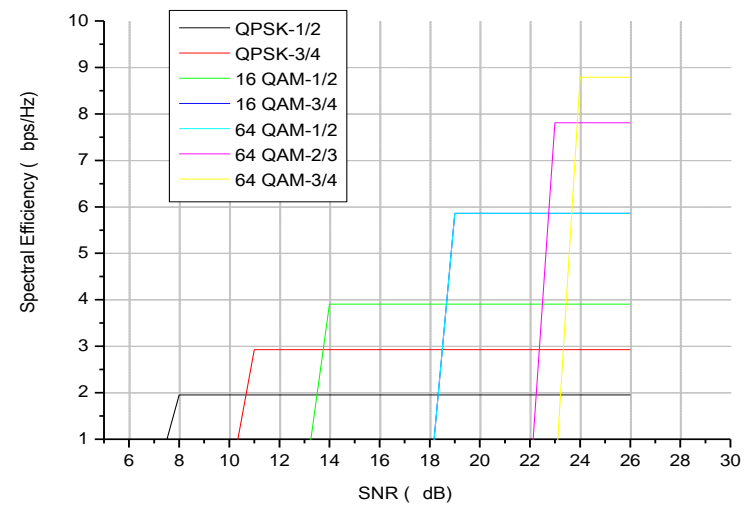

Figure 4.10 Spectral Efficiency versus SNR for individual MCSs over AWGN channel

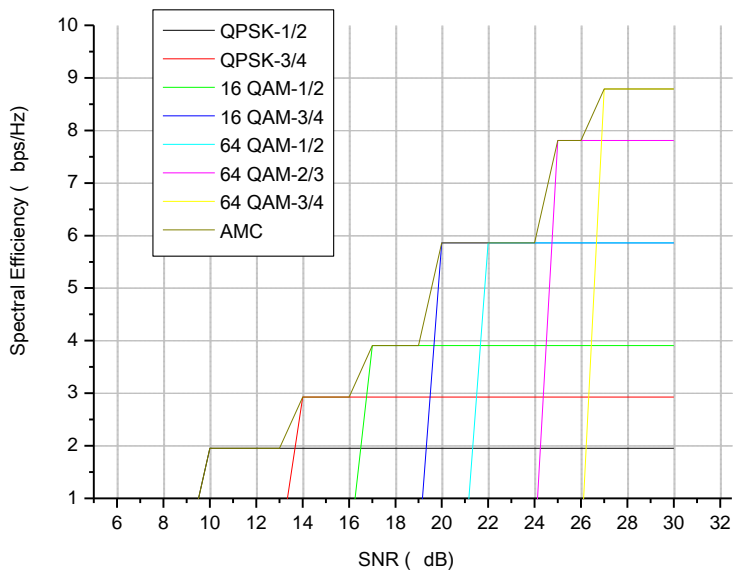

Figure 4.11 Spectral Efficiency versus SNR of AMC over Rician Flat fading channel

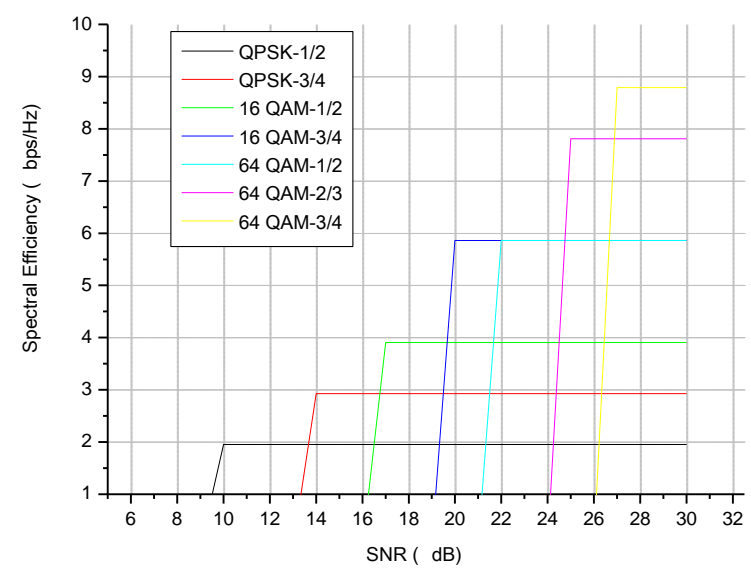

Figure 4.12 Spectral Efficiency versus SNR for individual MCSs over Rician Flat fading channel

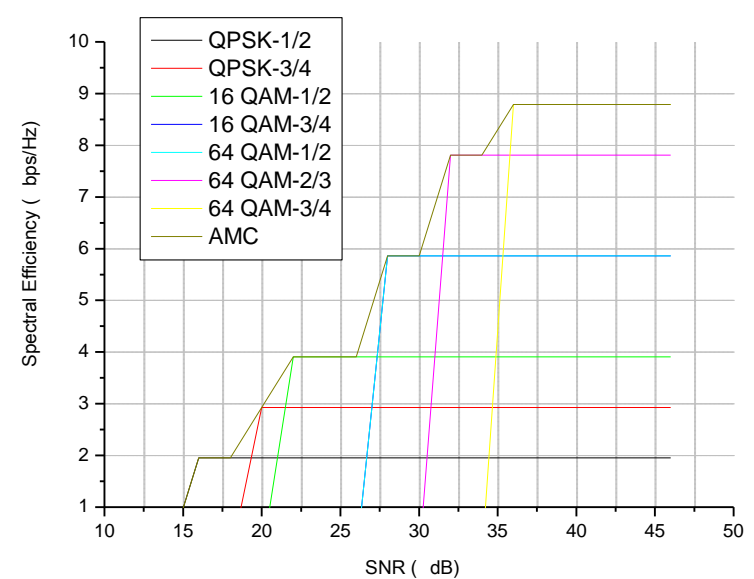

Figure 4.13 Spectral Efficiency versus SNR of AMC over Rician Frequency selective fading channel

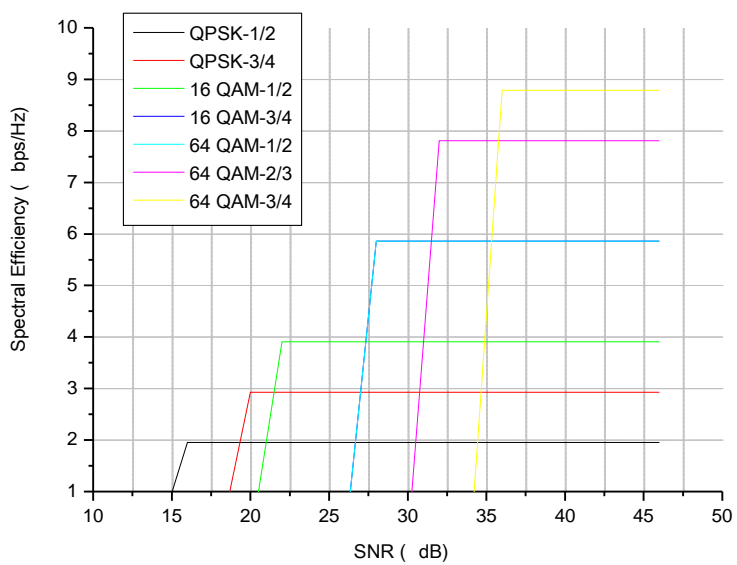

Figure 4.14 Spectral Efficiency versus SNR for individual MCSs over Rician Frequency selective fading channel

AMC clearly performed better than individual MCS during the analysis for the various fading channels as shown in the above figures $(4.11,4.12,4.13,4.14,4.15$ and 4.16). However, each fading channel performed differently and this is demonstrated in figure 4.17 showing the spectral efficiency for the various channels comparatively and summarized as follows: 


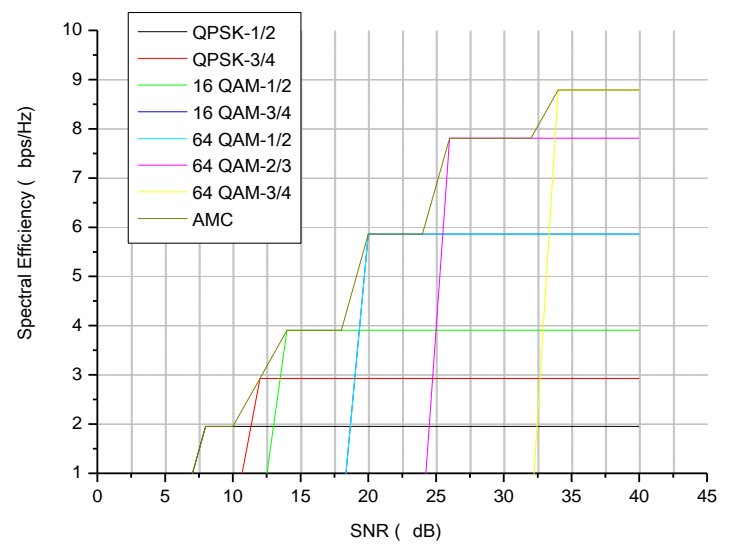

Figure 4.15 Spectral Efficiency versus SNR of AMC over Rayleigh fading channel

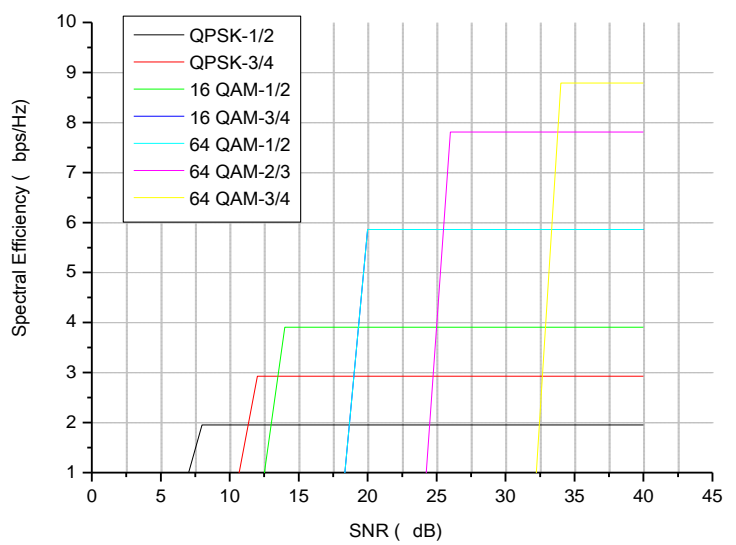

Figure 4.16 Spectral Efficiency versus SNR for individual MCSs over Rayleigh fading channel

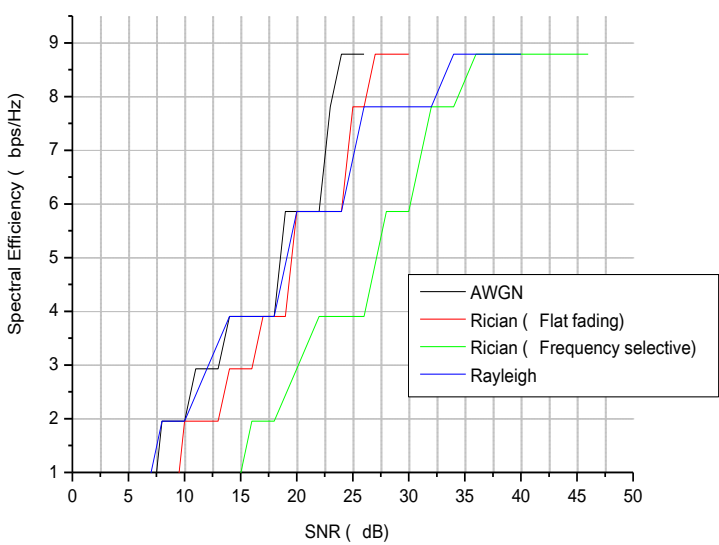

Figure 4.17 Spectral Efficiency versus AMC over various channels

AWGN channel provides better spectral efficiency than the fading channel (i.e. Rayleigh and Rician). Rayleigh interestingly gives almost the same spectral efficiency as AWGN at lower order modulation and coding rates but proceed to perform poorer at higher order modulation and coding rates. Rician Frequency selective fading channel offer the worst performance in terms of spectral efficiency compared to the other channels (fading and non-fading). Rician flat fading gives a better performance at high order modulation and coding rates but offer less desirable performance at lower order.

\section{CONCLUSION}

From the simulation results, it is realized that BER performance of transmission over non-fading AWGN channels improves with increasing SNR values as compared to that of the fading channels (Rician and Rayleigh). For lower MCSs like QPSK, Rayleigh channels perform better even at low SNR values than Rician channels. However, for high MCSs like 64QAM, Rayleigh performs poorer as against that of Rician channels. With Rician channels, flat fading perform better than frequency selective channels.

In terms of spectral efficiency, our results depict that AWGN channel provides better spectral efficiency than the fading channel (i.e. Rayleigh and Rician). However, it could also be realized that Rayleigh interestingly gives almost the same spectral efficiency as AWGN at lower order modulation and coding rates but proceed to offer a lesser performance at higher order modulation and coding rates. Rician Frequency selective fading channel offer the worst performance in terms of spectral efficiency compared to the other channels (fading and non-fading). Rician flat fading gives a better performance at high order modulation and coding rates but offer less desirable performance at lower order.

On signal power requirement, we concluded that the lower the MCS, the more efficient it is in terms of signal power. As per the result, QPSK-1/2 performed better at lower SNR values than the higher modulation and coding schemes in terms of BER. However, it offers the least bit rate presenting the least bandwidth making it suitable for long distance transmission. In case of data rate, 64QAM$3 / 4$ which is the highest MCS considered in this study offer the best data rate with excellent bandwidth utilization; however, require a high signal power for optimum performance compared to the others. 64QAM is however suitable for short distance transmission.

Employing AMC (Adaptive Modulation and Coding); each of these MCS is engaged in an instantaneous adjustment at each given time based on the channel quality, ensuring efficient utilization of bandwidth and signal power as well as enhancing capacity and overall throughput.

\section{REFERENCES}

[1] ITU-D. ITU-D Measuring the Information society 2013. ICT facts and figures 2013.

[2] ITU-D.ITU-D Measuring the information Society 2014. ICT facts and figures 2014.

[3] W. Weiwei. "Performance evaluation of inter-cell mitigation techniques for OFDMA cellular networks". 2010

[4] R. Prasad, J. Fernando and Velez. "OFDMA WiMAX Physical Layer in WiMAX Networks". Springer Netherlands, 2010

[5] R.Verma and G. Pankaj. "Interpretation of IEEE 802.16e (Wimax),"GJCST-E: Network, Web \& Security 13, no. 10 (2013).

[6] IEEE Standard for Local and metropolitan area networks Part 16: Air Interface for Broadband Wireless Access Systems Amendment 1: Multihop Relay Specification," IEEE Std 802.16j-2009 (Amendment to IEEE Std 802.16-2009), vol., no., pp.1,290, June 122009

[7] O. Sanida. "WiMAX overview." Faculty of Electrical Engineering, University of Ljubljana, Slovenia (2006). 
[8] C. Eklund, B. M. Roger, K. L. Stanwood and S. Wang. "IEEE standard 802.16: a technical overview of the WirelessMAN/sup $\mathrm{TM} /$ air interface for broadband wireless access," Communications Magazine, IEEE , vol.40, no.6, pp.98,107, June 2002

[9] B. M. Roger. "IEEE standard 802.16: a technical overview of the WirelessMANTM air interface for broadband wireless access." IEEE communications magazine (2002): 98.

[10] N. Kumar, D. Punetha and S. Singh. "Implementation of Soft Handover in IEEE 802.16 e." parameters 802: 802-16.

[11] Ramraj, Rajeshkumar, I. Ahmad, and D. Habibi. "Multi-Access Environments in Next Generation Networks." (2011).

[12] E. Ove, M. Sandell, J. Van De Beek, D. Landstrom, and F. Sjoberg. "An introduction to orthogonal frequency-division multiplexing."Lulea University of Technology, Sep. 1996

[13] J. Zhang, S. Feng, W Y and H. Zhuang, "MAC Performance Evaluation of IEEE 802.16j," Information Science and Engineering, 2008. ISISE '08. International Symposium on, vol.1, no., pp. $421,425,20-22$ Dec. 2008

[14] S. K. Wasan, I. Mahamod, N. Rosdiadee, and I. Shayea. "Thoughput Performance of Adaptive Modulation and Coding Scheme with Link Adaptation for Mimo-Wimax Downlink Transmission." JASR, no. 11 (2012): 641-650.

[15] H. Harald and B. Ghimire. "Introduction to Orthogonal Frequency Division Multiplexing (OFDM)." Advanced Digital Communication, The University of Edinburgh, 2009

[16] IEEE Standard 802.16-2009, "Part 16: Air Interface for Broadband Wireless Access Systems," May 2009.

[17] R. Cohen and G. Grebla, "Multi-dimensional OFDMA scheduling in a wireless network with relay nodes," INFOCOM, 2014

[18] S. Zhang, C. Zhu, J. K. O. Sin, and P. K. T. Mok, "A novel ultrathin elevated channel low-temperature poly-Si TFT", IEEE Electron Device Lett., vol. 20, pp. 569-571, Nov. 1999. 